\title{
PET Study of Sphingosine-1-phosphate Receptor 1 Expression in Response to $S$. aureus Infection
}

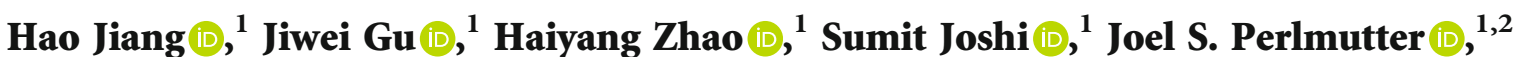 \\ Robert J. Gropler $\mathbb{D}^{1},{ }^{1}$ Robyn S. Klein $\mathbb{D}^{2,3,4}$ Tammie L. S. Benzinger ${ }^{(\mathbb{D})}{ }^{1,5}$ and Zhude Tu ${ }^{1}{ }^{1}$ \\ ${ }^{1}$ Department of Radiology, Washington University School of Medicine, St Louis, MO 63110, USA \\ ${ }^{2}$ Department of Neuroscience, Neurology, Washington University School of Medicine, St Louis, MO 63110, USA \\ ${ }^{3}$ Department of Medicine, Washington University School of Medicine, St Louis, MO 63110, USA \\ ${ }^{4}$ Department of Pathology and Immunology, Washington University School of Medicine, St Louis, MO 63110, USA \\ ${ }^{5}$ Department of Neurological Surgery, Washington University School of Medicine, St Louis, MO 63110, USA
}

Correspondence should be addressed to Zhude Tu; zhudetu@wustl.edu

Received 28 May 2021; Accepted 2 September 2021; Published 4 October 2021

Academic Editor: Ali Azhdarinia

Copyright (c) 2021 Hao Jiang et al. This is an open access article distributed under the Creative Commons Attribution License, which permits unrestricted use, distribution, and reproduction in any medium, provided the original work is properly cited.

Sphingosine-1-phosphate receptor 1 (S1PR1) plays a crucial role in infectious diseases. Targeting S1PR1 provides protection against pathogens, such as influenza viruses. This study is aimed at investigating S1PR1 in response to bacterial infection by assessing S1PR1 expression in S. aureus-infected mice. A rodent local muscle bacterial infection model was developed by injecting S. aureus to the lower hind limb of Balb/c mice. The changes of S1PR1 expression in response to bacterial infection and blocking treatment were assessed using ex vivo biodistribution and in vivo positron emission tomography (PET) after intravenous injection of an S1PR1-specific radiotracer $\left[{ }^{18} \mathrm{~F}\right] \mathrm{TZ} 4877$. The specificity of $\left[{ }^{18} \mathrm{~F}\right] \mathrm{TZ} 4877$ was assessed using S1PR1specific antagonist, NIBR-0213, and S1PR1-specific DsiRNA pretreated the animals. Immunohistochemical studies were performed to confirm the increase of S1PR1 expression in response to infection. Ex vivo biodistribution data showed that the uptake of $\left[{ }^{18} \mathrm{~F}\right] \mathrm{TZ} 4877$ was increased $30.6 \%, 54.3 \%, 74.3 \%$, and $115.3 \%$ in the liver, kidney, pancreas, and thymus of the infected mice, respectively, compared to that in normal control mice, indicating that S1PR1 is involved in the early immune response to bacterial infection. NIBR-0213 or S1PR1-specific DsiRNA pretreatment reduced the tissue uptake of $\left[{ }^{18} \mathrm{~F}\right] \mathrm{TZ} 4877$, suggesting that uptake of $\left[{ }^{18} \mathrm{~F}\right] \mathrm{TZ} 4877$ is specific. Our PET/CT study data also confirmed that infected mice have increased $\left[{ }^{18} \mathrm{~F}\right]$ TZ4877 uptake in several organs comparing to that in normal control mice. Particularly, compared to control mice, a $39 \%$ increase of $\left[{ }^{18} \mathrm{~F}\right] \mathrm{TZ} 4877$ uptake was observed in the infected muscle of $S$. aureus mice, indicating that S1PR1 expression was directly involved in the inflammatory response to infection. Overall, our study suggested that S1PR1 plays an important role in the early immune response to bacterial infection. The uptake of $\left[{ }^{18} \mathrm{~F}\right] \mathrm{TZ} 4877$ is tightly correlated with the S1R1 expression in response to $S$. aureus infection. PET with S1PR1-specific radiotracer $\left[{ }^{18} \mathrm{~F}\right] \mathrm{TZ} 4877$ could provide a noninvasive tool for detecting the early S1PR1 immune response to infectious diseases.

\section{Introduction}

Staphylococcus aureus (S. aureus) is a commensal human microbiota with approximately $30 \%$ of the human population which is colonized with $S$. aureus [1]. S. aureus often causes skin and soft tissue infections but can also cause pneumonia, bloodstream infections, endocarditis, and osteomyelitis [2-4]. To date, with the majority of studies focused on virus infection, particularly influenza, only little is known about the role of sphingosine-1-phosphate (S1P) and S1P receptors (S1PRs) in response to bacterial infection. Igawa and colleagues demonstrated that the expression of S1PR2 was elevated in S. aureus-treated human epidermal keratinocytes; they also showed that both S1PR1 and S1PR2 controlled the proinflammatory cytokine expression and secretion during the S. aureus invasion [5].

S1P binds to specific G protein-coupled S1P receptors 15 and triggers the S1P/S1PR pathway which plays a 
regulatory role in many pathophysiological processes, including angiogenesis [6], neurodegeneration [7], and immune response [8]. Intriguingly, it has been reported that S1P signaling via S1PRs is related to various aspects of inflammatory cell function. Accumulated evidence suggests that the S1P/S1PR pathway is impaired during infectious diseases. Furthermore, the S1P/S1PR pathway also plays an important role in infection-induced sepsis [9-11]. As a consequence, the S1P/S1PR pathway has been widely accepted as a therapeutic target in inflammatory and infection diseases [12]. Of note, several multiple sclerosis (MS) disease-modifying therapies targeting S1P/S1PRs are under investigation for treating coronavirus disease-2019 (COVID-19) [13, 14].

Out of the five S1PRs, S1PR1 is the most abundant S1P receptor and is widely studied in different diseases. It is ubiquitously expressed among tissues and is heavily involved in immune cell regulation. S1PR1 has been found to mediate functions in most immune cells and therefore plays a curial role in both innate and adaptive immune responses [15]. Previous studies suggest that S1PR1 is essential for lymphocyte recirculation by regulating lymphocyte egress from both the thymus and peripheral lymphoid organs [16, 17]; activation of S1PR1 inhibits the migration of T lymphocytes into different lymphatics and results in retention of T lymphocytes in nonlymphoid peripheral tissues [18]. S1PR1 also regulates the migration of $\mathrm{B}$ lymphocytes and osteoclasts $[19,20]$. It was reported that S1PR1 plays an important role in the immune response to infectious diseases by regulating recruitment and trafficking of innate immune cells, macrophage polarization [21], and dendritic cell functions [22]. In addition, S1PR1 is considered to play a critical role in the development of sepsis [11]; for example, S1PR1-specific agonist SEW2871 can successfully protect against renal injury in a sepsis model [23]. Numerous studies show that S1PR1 expression or activation is tightly correlated with the inflammatory response to infectious diseases such as Newcastle disease virus infection [24], influenza A virus H1N1 [25], H9N2 infection [26], herpes simplex virus type 1 infection [27], Pseudomonas aeruginosa lung infection [28], and human immunodeficiency viruses HIV-1 infection [29]. It is also believed that host-directive therapy using modulators of S1PR1 and other S1PRs may be an effective treatment against severe infectious diseases, such as severe acute respiratory syndrome (SARS) and COVID-19 [30]. Despite the current understanding of S1PR1 in normal lymphocyte trafficking, the precise mechanisms of S1PR1 activation in response to pathogen-derived pathological infectious consequences remain not clear.

Our previous positron emission tomography (PET) studies with S1PR1-specific tracer demonstrated an increased expression of S1PR1 in rodent models of vascular inflammation and neuroinflammation [31, 32]. To evaluate the ability of S1PR1-specific tracer for the assessment of other inflammatory diseases, as well as to further understand the role of S1PR1 in response to pathogen infection, we performed ex vivo biodistribution, microPET, and immunohistochemistry staining studies to investigate S1PR1 expression on $S$. aureus-infected mice using our recently reported S1PR1-specific radiotracer [ [18]F]TZ4877 [33-35]. In our studies, we observed an increased uptake of $\left[{ }^{18} \mathrm{~F}\right] \mathrm{TZ} 4877$ in several organs in response to the infection; a $39 \%$ increase of $\left[{ }^{18} \mathrm{~F}\right] \mathrm{TZ} 4877$ uptake was also observed at the local hind limb muscle infected site. Our results indicated that the infection-induced increase of S1PR1 expression is tightly correlated with pathogen-derived inflammation. Our findings are consistent with other studies that S1PR1 plays a critical role in response to pathogen infection.

\section{Materials and Methods}

2.1. Animals. All animal experiments were conducted by following the Guidelines for the Care and Use of Research Animals under a research protocol approved by Washington University Institutional Animal Care and Use Committee (IACUC). Biodistribution and microPET/CT studies were conducted in Preclinical Imaging Facility at Washington University School of Medicine in St. Louis. For infection model, mice were injected with bacteria or PBS under 2$3 \%$ isoflurane in $\mathrm{O}_{2}$. For biodistribution study, mice were euthanized by cervical dislocation under anesthesia with 2$3 \%$ isoflurane in $\mathrm{O}_{2}$ and then, tissues of interest were collected. For microPET/CT studies, mice were euthanized by $\mathrm{CO}_{2}$ inhalation after use.

2.2. Radiosynthesis. S1PR1-specific radiotracer $\left[{ }^{18} \mathrm{~F}\right] \mathrm{TZ} 4877$ was used in this study in order to detect changes of S1PR1 in response to $S$. aureus infection. The synthesis and quality control of $\left[{ }^{18} \mathrm{~F}\right] \mathrm{TZ} 4877$ were achieved as previously reported (Figure 1(a)) [33]. For each batch, the synthesis of $\left[{ }^{18} \mathrm{~F}\right] \mathrm{TZ} 4877$ was accomplished with a radiochemical purity of $>99 \%$, chemical purity of $>95 \%$, and molar activity of $>48 \mathrm{GBq} / \mu \mathrm{mol}$ (decay corrected to the end of synthesis, EOB).

2.3. Bacterial Cell Culture. Staphylococcus aureus (ATCC 29213) was purchased from the American Type Culture Collection. The stock $S$. aureus was plated on LB agar plates overnight; individual colony was picked from LB agar plate and cultured in Trypticase Soy Broth at $37^{\circ} \mathrm{C}$ and $210 \mathrm{rpm}$ until approximately $10 \times 10^{8} / \mathrm{mL}$ of bacterial concentration was reached. The bacterial concentration was measured using a DEN-1 densitometer (Grant Instruments, UK). S. aureus was then centrifuged and resuspended in $25 \mu \mathrm{L}$ of PBS at a colony-forming unit (CFU) of $1 \times 10^{8} \mathrm{CFU}$ of live bacteria for high titer and $1 \times 10^{6} \mathrm{CFU}$ of live bacteria for low titer and used immediately.

2.4. S. aureus Infection through Local Inoculation. All experimental procedures involving animals were performed according to guidelines established by the Animal Studies Committee at Washington University in St. Louis. Sevenweek-old Balb/c mice (Charles River, Wilmington, MA) were used in all studies. All animals were randomly assigned to each study group. In order to evaluate the effect of $S$. aureus infection on S1PR1, a bacterial infection model was created following the previously described procedure [36-38]. Previous studies demonstrated that S1PR1 is significantly increased 24 hours after Newcastle disease virus infection; in order to detect the change of S1PR1 expression using PET imaging, a relatively high dose of $S$. aureus and 


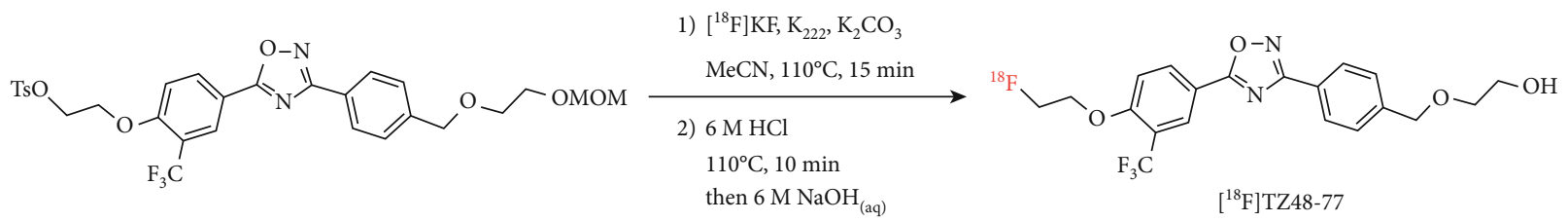

(a)
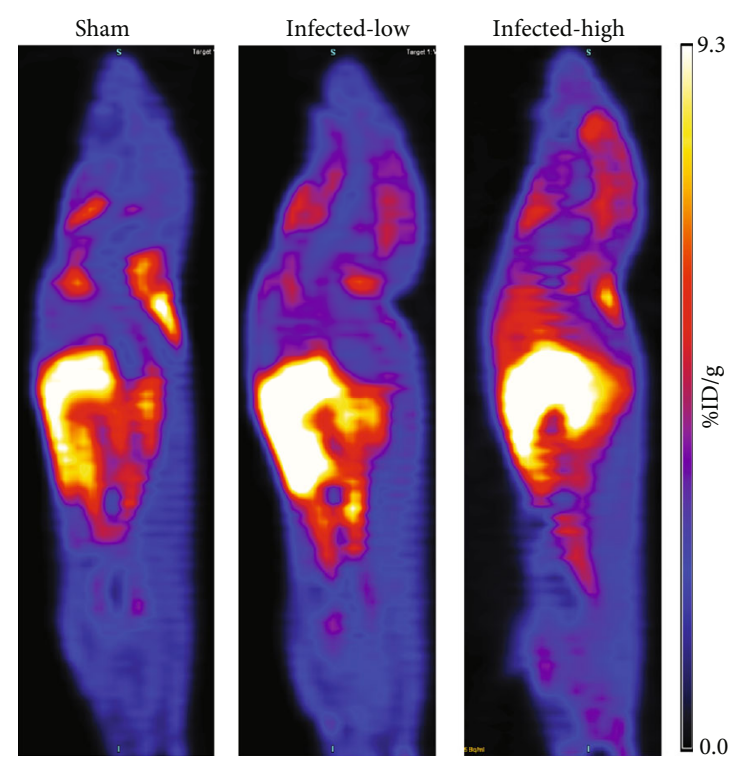

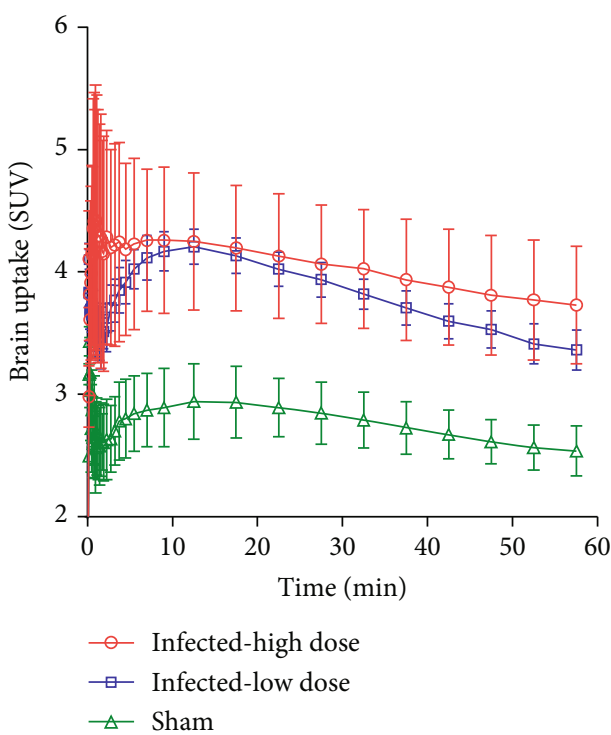

(c)

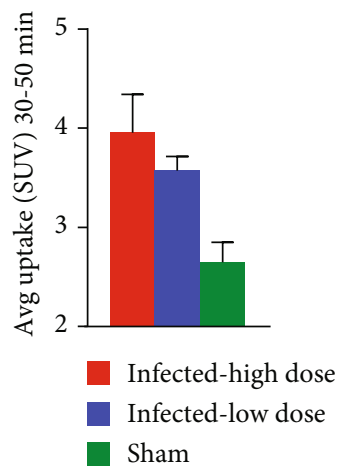

(d)

FIGURE 1: MicroPET imaging of S1PR1 activity in S aureus-infected mice. (a) Radiosynthesis of S1PR1-specific radiotracer, $\left[{ }^{18} \mathrm{~F}\right] \mathrm{TZ} 4877$; (b) representative sagittal microPET images of $\left[{ }^{18} \mathrm{~F}\right] \mathrm{TZ} 4877$ in mice. Comparing with sham mice, the tracer uptake was significantly higher in the infected mice, and the increased uptake of the tracer showed $S$ aureus dose dependent; (c) the tracer uptake in the brain was quantified; time-activity curves showed that the tracer uptake in infected mice was significantly higher than mice without infections; (d) the average tracer uptake in the brain from 30 to $50 \mathrm{~min}$ of the PET scan showed a dose-dependent manner. Data represent the mean \pm SEM, $n=3$ for each group.

24-hour treatment were used for initial evaluation of the response of S1PR1 to the bacterial infection. In fact, it has been reported that 10 [7] to 10 [9] CFU S. aureus can cause bacteria to elicit inflammatory responses within 24 hours [39-41], and intravenous inoculation of $5 \times 10^{7}$ to $5 \times 10^{8}$ CFU $S$. aureus can cause the animal to develop septic shock within 48 hours [42]. In our case, $1 \times 10^{8} \mathrm{CFU}$ (high titer) and $1 \times 10^{6} \mathrm{CFU}$ (low titer) of live $S$. aureus were diluted in $25 \mu \mathrm{L}$ PBS and injected in the muscle of the lower hind limb 24 hours prior to the study; $25 \mu \mathrm{L}$ of sterilized PBS was also injected to the contralateral limb as an internal control; in the meantime, a group of sham animals received the same volume of sterile PBS. For blocking studies, either S1PR1-specific ligand NIBR-0213 (Sigma-Aldrich, St. Louis, $\mathrm{MO})$ at a dose of $5 \mathrm{mg} / \mathrm{kg}$ or S1PR1-specific DsiRNA (mm.Ri.S1pr1.13, Integrated DNA Technologies, Coralville, IA) at a dose of $20 \mu \mathrm{g} / \mathrm{kg}$ was administered 12 hours prior to injection of radiotracer, noticing that NIBR-0213 has a long elimination half-life and prolonged duration of action in rodent [43]. In addition, our initial pilot test demonstrated that NIBR-0213 can successfully block our radiotracer long time after treatment.

2.5. Ex Vivo Biodistribution Studies. To characterize the distribution and kinetics of $\left[{ }^{18} \mathrm{~F}\right] \mathrm{TZ} 4877$ in different tissues in normal mice, we performed an ex vivo biodistribution study in normal Balb/c mice as previously described with minor modifications [33]. In brief, approximately $2.2 \mathrm{MBq} / 100 \mu \mathrm{L}$ of the radiotracer was administrated to the mice intravenously via the tail vein. At 5, 30, or $60 \mathrm{~min}$ posttracer injection, animals were euthanized and tissues of interest including the blood, lung, liver, spleen, kidney, muscle, heart, brain, pancreas, thymus, and small intestine were collected, weighed, and counted in a Beckman 8000 automated gamma counter (Beckman, Brea, CA). The radiotracer uptake in each tissue was calculated as background and decay-corrected percent injected dose per gram $(\% \mathrm{ID} / \mathrm{g})$. 
To determine the radiotracer uptake in different tissues in response to $S$. aureus infection, we then performed an ex vivo biodistribution study in normal and $S$. aureusinfected Balb/c mice. After 24 hours of infection through local inoculation, biodistribution study was performed as described above, tissues of interest from sham and infected mice were collected $30 \mathrm{~min}$ posttracer injection, and the uptake in each tissue was calculated. To determine if the increased uptake of $\left[{ }^{18} \mathrm{~F}\right] \mathrm{TZ} 4877$ was caused by endogenous S1PR1 activation, the infected mice were pretreated with an S1PR1-specific antagonist, NIBR-0213 [43], at $5 \mathrm{mg} / \mathrm{kg}$ and 12 hours prior to tracer injection. In addition, we used S1PR1-specific DsiRNA at $20 \mu \mathrm{g} / \mathrm{kg}$ pretreatment in mice 12 hours prior to tracer injection to further confirm that $\left[{ }^{18} \mathrm{~F}\right] \mathrm{TZ} 4877$ is specific to S1PR1 in vivo, rather than other S1P receptor subtypes. The infected mice received $1 \times 10^{6}$ CFU of live $S$. aureus that was diluted in $25 \mu \mathrm{L}$ PBS and injected into the muscle of the lower hind limb 24 hours prior to the study.

2.6. Immunohistochemistry. To confirm the increased uptake of $\left[{ }^{18} \mathrm{~F}\right] \mathrm{TZ} 4877$ in $S$. aureus-infected mice is caused by the upregulation of S1PR1, immunohistochemistry staining was performed in hind limb muscle from sham and $S$. aureus-infected mice. 14-micron sections from fresh frozen tissue were used. Sections were prewarmed to room temperature and fixed with 4\% paraformaldehyde in PBS for 15 minutes and then washed in PBS. Sections were then incubated in Antigen Retrieval Buffer (Abcam, Cambridge, MA) for 20 minutes in boiling water bath and then blocked with $5 \%$ horse serum for 2 hours at room temperature followed by blocking with ReadyProbes Endogenous HRP and AP Blocking Solution (ThermoFisher, Waltham, MA). After washing in PBS, all sections were then incubated with rabbit anti-S1PR1 (Alomone, Israel) antibody overnight at $4^{\circ} \mathrm{C}$ and then washed and incubated with ImmPRESS HRP Horse anti-rabbit polymer for 1 hour at room temperature and developed with ImmPACT DAB (Vector Laboratories, Burlingame, CA).

2.7. MicroPET/CT Studies. To confirm the changes of S1PR1 expression in response to $S$. aureus inoculation, as well as to evaluate the feasibility of our S1PR1-specific radiotracer in detecting the infection-induced S1PR1 activation, microPET/CT studies of $\left[{ }^{18} \mathrm{~F}\right] \mathrm{TZ} 4877$ were performed in normal controls and $S$. aureus-infected mice. For the microPET studies, an Inveon MM PET/CT scanner (Siemens, Germany) was used; mice were anesthetized with $2 \%$ isoflurane under gas anesthesia during the imaging data collection period. Mice were secured using a custom-designed acrylic restraining device. Following a transmission scan and a computed tomography for anatomical registration, 6.8$7.6 \mathrm{MBq} /$ mouse of $\left[{ }^{18} \mathrm{~F}\right] \mathrm{TZ} 4877$ was administrated via the tail vein using a catheter for injection. A list-mod protocol was used with 60-minute dynamic data acquisition with a dynamic sequence of $1 \times 3 \mathrm{~s}, 6 \times 2 \mathrm{~s}, 9 \times 5 \mathrm{~s}, 6 \times 10 \mathrm{~s}, 4 \times 30$ s, $2 \times 1 \mathrm{~min}, 2 \times 2 \mathrm{~min}$, and $10 \times 5 \mathrm{~min}$ frames. PET image data was processed and analyzed using Inveon Research Workstation software IRW 4.2 (Siemens, Germany). The data was reconstructed per time frame using an interactive reconstruction algorithm and corrected for decay. The tissue uptake of the radioactivity was measured from elliptical ROIs, and percent injected dose per gram of tissue (\%ID/g) was calculated. To determine if the uptake of $\left[{ }^{18} \mathrm{~F}\right] \mathrm{TZ} 4877$ was correlated with the severity of the infection, microPET studies were also performed in mice that were injected with a high dose of live $S$. aureus $\left(1 \times 10^{8} \mathrm{CFU}\right.$ of live $S$. aureus $)$.

2.8. Statistical Analysis. All data were analyzed with Prism 7.0 (GraphPad Software, San Diego, CA). Two-way ANOVA and paired Student $t$-test were used for comparison of percent injected dose per gram of tissue from ROI for PET study; two-way ANOVA with a relatively powerful Fisher LSD multiple comparisons test was used for comparison of percent injected dose per gram of tissue from each organ among different sample groups for the tissue distribution analysis. A $P$ value $\leq 0.05$ was considered to be statistically significant.

\section{Results}

3.1. Biodistribution Studies in Normal and S. aureus-Infected Mice. We first evaluated the kinetics of $\left[{ }^{18} \mathrm{~F}\right] \mathrm{TZ} 4877$ in mice at 5,30 , and $60 \mathrm{~min}$ postinjection (Table 1). The initial tracer uptake was high in tissues at $5 \mathrm{~min}$; a rapid clearance of radioactivity was observed in the majority of tested tissues including the blood, lung, liver, spleen, kidney, muscle, heart, brain, pancreas, and thymus from $5 \mathrm{~min}$ to $60 \mathrm{~min}$. Tracer uptake gradually accumulated in the small intestine, suggesting it had hepatobiliary clearance. In general, at $30 \mathrm{~min}$ postinjection in normal $\mathrm{Balb} / \mathrm{c}$ mice, the muscle had the lowest tracer uptake among the tissues evaluated with a $\% \mathrm{ID} / \mathrm{g}$ of $1.4 \pm 0.18$, whereas the mouse liver and small intestine had the highest tracer uptake level with $\% \mathrm{ID} / \mathrm{g}$ of $15.27 \pm 0.63$ and $11.73 \pm 0.59$. Notably, $\left[{ }^{18} \mathrm{~F}\right] \mathrm{TZ} 4877$ showed a relatively high mouse brain uptake with \%ID/g of $4.15 \pm 0.19$ at $30 \mathrm{~min}$ postinjection, indicating that $\left[{ }^{18} \mathrm{~F}\right] \mathrm{TZ} 4877$ penetrates the blood-brain barrier well.

We next compared the tissue uptake of $\left[{ }^{18} \mathrm{~F}\right] \mathrm{TZ} 4877$ between normal and S. aureus-infected mice. Interestingly, in response to $S$. aureus infection, an increase uptake of $\left[{ }^{18} \mathrm{~F}\right] \mathrm{TZ} 4877$ in several tissues was observed. After $30 \mathrm{~min}$ postinjection, the uptake of $\left[{ }^{18} \mathrm{~F}\right] \mathrm{TZ} 4877$ in serval organs including the lung, liver, spleen, brain, heart, kidney, thyroid, pancreas, thymus, and small intestine of infected mice was higher than that of sham mice (Table 2; ANOVA: $F(1,60)=54.6, P<0.0001)$. Fisher's LSD test following ANOVA showed that the tracer uptake in several tissues was significantly higher in mice with infections including the liver $(P=0.002 ; 30.6 \%$ of increase), kidney $(P=0.0031$; $54.3 \%$ of increase), pancreas ( $P=0.0027 ; 74.3 \%$ of increase), thymus ( $P=0.0006 ; 115.3 \%$ of increase), and small intestine $(P<0.0001 ; 40.0 \%$ of increase).

In addition, the $S$. aureus-induced increase uptake of $\left[{ }^{18} \mathrm{~F}\right] \mathrm{TZ} 4877$ can be blocked by NIBR-0213 pretreatment and S1PR1-specific DsiRNA pretreatment. In general, NIBR-0213 significantly reduced the uptake of $\left[{ }^{18} \mathrm{~F}\right]$ TZ4877 in several organs of the infected mice 
TABLE 1: Biodistribution (\%ID/g, mean \pm SEM) of S1PR1-specific $\left[{ }^{18} \mathrm{~F}\right] \mathrm{TZ} 4877$ in Balb/c mice $(n=4)$.

\begin{tabular}{lccc}
\hline & $5 \mathrm{~min}$ & $30 \mathrm{~min}$ & $60 \mathrm{~min}$ \\
\hline Blood & $3.78 \pm 0.18$ & $2.53 \pm 0.08$ & $2.68 \pm 0.03$ \\
Lung & $8.81 \pm 0.44$ & $5.8 \pm 0.23$ & $5.18 \pm 0.08$ \\
Liver & $20.07 \pm 0.17$ & $15.27 \pm 0.63$ & $17.29 \pm 0.19$ \\
Spleen & $4.94 \pm 0.24$ & $3.28 \pm 0.17$ & $2.92 \pm 0.01$ \\
Kidney & $10.47 \pm 0.35$ & $7.34 \pm 0.45$ & $6.57 \pm 0.09$ \\
Muscle & $1.76 \pm 0.37$ & $1.4 \pm 0.18$ & $1.53 \pm 0.08$ \\
Heart & $7.26 \pm 0.26$ & $4.39 \pm 0.24$ & $4.03 \pm 0.13$ \\
Brain & $4.96 \pm 0.36$ & $4.15 \pm 0.19$ & $3.78 \pm 0.09$ \\
Pancreas & $8.27 \pm 0.51$ & $5.42 \pm 0.3$ & $4.75 \pm 0.19$ \\
Thymus & $7.48 \pm 0.68$ & $5.61 \pm 0.39$ & $6.24 \pm 0.56$ \\
Small intestine & $8.06 \pm 0.27$ & $11.73 \pm 0.59$ & $17.92 \pm 1.06$ \\
\hline
\end{tabular}

(Table 2; ANOVA between infected vs. infected with NIBR0213: $F(1,60)=22.7, P<0.0001)$. Fisher's LSD test following ANOVA showed that the uptake of $\left[{ }^{18} \mathrm{~F}\right] \mathrm{TZ} 4877$ was significantly decreased after NIBR-0213 pretreatment in the liver ( $P=0.0002 ; 22.5 \%$ of decrease), kidney $(P=0.0225$; $30.7 \%$ of decrease), and small intestine $(P=0.0011 ; 12.0 \%$ of decrease). Furthermore, similar to the treatment with S1PR1 antagonist, S1PR1-specific DsiRNA also significantly reduced the tracer uptake in the liver and small intestine of the infected mice to the uptake level of $\left[{ }^{18} \mathrm{~F}\right] \mathrm{TZ} 4877$ in sham mice (Table 2; ANOVA between infected vs. infected with DsiRNA: $F(1,60)=15.24, P=0.0002)$. Fisher's LSD test following ANOVA showed that the tracer uptake was significantly restored after DsiRNA treatment in the liver $(P=0.0035 ; 15.4 \%$ of decrease $)$ and small intestine $(P<0.0001 ; 7.8 \%$ of decrease $)$.

3.2. MicroPET Studies in Normal and S. aureus-Infected Mice. In vivo microPET studies were carried out in three groups of mice, a low-dose inoculation of bacteria $\left(1 \times 10^{6}\right.$ $\mathrm{CFU})$, a high-dose inoculation of bacteria $\left(1 \times 10^{8} \mathrm{CFU}\right)$, and sterile PBS buffer. Similar to biodistribution results, a systemic increased uptake of $\left[{ }^{18} \mathrm{~F}\right] \mathrm{TZ} 4877$ was identified in microPET studies. The radiotracer uptake in the liver and brain, as well as several other tissues, was significantly increased in the infected mice. Interestingly, the uptake of $\left[{ }^{18} \mathrm{~F}\right] \mathrm{TZ} 4877$ in these organs showed a dose-dependent manner to the S. aureus infection (Figure 1(b), Supplemental Figures 1 and 3). For example, tissue time-activity curves showed that the brain uptake in the infected mice was significantly higher than the uptake in sham mice in a dosedependent manner (Figure 1(c); ANOVA test: high vs. low: $F(1,120)=9.62, P=0.0024$; high vs. sham: $F(1,80)=95.6$, $P<0.001$; low vs. sham: $F(1,120)=337.2, P<0.001)$. Comparing with sham mice, though the difference between high and low dose of infection was not statistically different, the uptake of $\left[{ }^{18} \mathrm{~F}\right] \mathrm{TZ} 4877$ in infected mice from 30 to $50 \mathrm{~min}$ of the scan was $\sim 49.4 \%$ higher in the high-dose group and $\sim 34.8 \%$ higher in the low-dose group (Figure 1(d)).
MicroPET study showed that the uptake of $\left[{ }^{18} \mathrm{~F}\right] \mathrm{TZ} 4877$ in the hind limb muscle was relatively low with an SUV of $\sim 1.5$ in sham mice. Interestingly, though the uptake of $\left[{ }^{18} \mathrm{~F}\right] \mathrm{TZ} 4877$ was low, compared with sham mice, the uptake of $\left[{ }^{18} \mathrm{~F}\right] \mathrm{TZ} 4877$ in the hind limb of $S$. aureus-infected mice was significantly higher than that of the sham mice. The average tracer uptake in the hind limb muscle from 30 to 50 min of the PET scan showed a $\sim 39 \%$ increase uptake (SUV) of $\left[{ }^{18} \mathrm{~F}\right] \mathrm{TZ} 4877$ in infected mice compared to that in the normal control mice with a $P$ value of 0.0083 (Figures 2(a)-2(c)). Notably, the increase of tracer uptake in the muscle was higher than the increase in the brain and liver of the infected mice (Table 3). Moreover, the increase of $\left[{ }^{18} \mathrm{~F}\right] \mathrm{TZ} 4877$ uptake was only identified in the ipsilateral side of the infection site but not in the contralateral site (Supplemental Figures 2 and 3). In contrast, after 24 hours of inoculation, the expression of S1PR1 measured by PET with $\left[{ }^{18} \mathrm{~F}\right] \mathrm{TZ} 4877$ showed almost no uptake in the hind limb muscle of the high-dose inoculation group (Supplemental Figure 1).

3.3. Immunohistochemistry Analysis in S. aureus-Infected Mice. Immunohistochemistry analysis was carried out in the hind limb muscle of mice with and without infection. After 24 hours of $S$. aureus infection, the expression level of S1PR1 was significantly elevated in the muscle of infected mice in agreement with the increased uptake of $\left[{ }^{18} \mathrm{~F}\right] \mathrm{TZ} 4877$ (Figure 3), indicating that the upregulation of S1PR1 was tightly correlated with the pathogen-derived inflammation.

\section{Discussion}

Increasing evidence indicates that S1PR1 expression is tightly correlated with the inflammatory response to infectious diseases such as viral infections [44-49], bacterial infections [50-55], protozoan infection [56], and fungal infection $[57,58]$. It is believed that S1PR1 plays an important role in maintaining endothelial barrier function and integrity in normal as well as inflammatory conditions and can modulate the endothelium to suppress inflammatory responses [59].

Mouse models for infectious diseases caused by $S$. aureus have been widely used for the evaluation of $S$. aureus skin and soft tissue infection, bacteremia, sepsis, peritonitis, and endocarditis [3]. Immune-competent mice, such as C57/BL6 and Balb/c mice, are considered a good candidate for the study of S. aureus infection in soft tissue [3]. The primary aim of this study was to evaluate if there is a change of S1PR1 expression in response to $S$. aureus infection and if such infection-induced changes of S1PR1 can be detected by our S1PR1-specific radiotracer $\left[{ }^{18} \mathrm{~F}\right] \mathrm{TZ} 4877$. Therefore, a relatively high dose of $S$. aureus, $1 \times 10^{6} \mathrm{CFU}$, was used to induce the infection, and an even higher dose at $1 \times 10^{8}$ CFU was used to compare if the changes of S1PR1 were $S$. aureus dose dependent. In the meantime, in order to detect the local and systemic changes of S1PR1, a relatively long inoculation period of 24 hours was chosen. In fact, it has been well known that soft tissue infection with 10 [7] to 10 [9] CFU S. aureus can cause bacteria to elicit inflammatory 
TABLE 2: Biodistribution of S1PR1-specific $\left[{ }^{18} \mathrm{~F}\right] \mathrm{TZ} 4877$ in sham, infected, and infected with treatments mice $(n=4)$.

\begin{tabular}{lcccc}
\hline & Sham & Infected & NIBR0213 & siRNA \\
\hline Blood & $2.33 \pm 0.17$ & $1.96 \pm 0.81$ & $2.60 \pm 0.1$ & $2.46 \pm 0.12$ \\
Lung & $5.56 \pm 0.18$ & $6.68 \pm 0.47$ & $5.67 \pm 0.22$ & $5.57 \pm 0.22$ \\
Liver & $13.52 \pm 1.29$ & $17.65 \pm 0.16^{* *}$ & $14.42 \pm 0.46^{\# \# \#}$ & $13.6 \pm 0.21^{\# \#}$ \\
Spleen & $2.39 \pm 0.17$ & $3.48 \pm 0.19$ & $3.17 \pm 0.12$ & $3.03 \pm 0.11$ \\
Brain & $3.36 \pm 0.27$ & $5.24 \pm 0.12$ & $4.55 \pm 0.17$ & $4.28 \pm 0.13$ \\
Heart & $3.56 \pm 0.37$ & $4.84 \pm 0.16$ & $4.20 \pm 0.14$ & $4.09 \pm 0.19$ \\
Kidney & $5.83 \pm 0.39$ & $8.99 \pm 0.32^{*}$ & $7.31 \pm 0.18^{\#}$ & $6.61 \pm 0.27$ \\
Thyroid & $2.83 \pm 0.15$ & $4.00 \pm 0.12$ & $3.29 \pm 0.09$ & $3.20 \pm 0.25$ \\
Pancreas & $4.33 \pm 0.34$ & $7.55 \pm 0.54^{*}$ & $6.10 \pm 0.5$ & $5.83 \pm 0.85$ \\
Thymus & $3.23 \pm 0.18$ & $6.94 \pm 1.02^{*}$ & $5.79 \pm 0.76$ & $16.31 \pm 2.13^{\# \#}$ \\
Small intestine & $14.96 \pm 1.12$ & $20.94 \pm 1.60^{* * * *}$ &
\end{tabular}

Data represents \%ID/g and mean \pm SEM, samples with a statistical difference were in italic; ${ }^{*}$ Fisher's LSD multiple comparisons between sham and infected mice: ${ }^{*} P<0.05,{ }^{* *} P<0.01$, and ${ }^{* * * *} P<0.0001$; ${ }^{*}$ Fisher's LSD multiple comparisons between infected and infected with NIBR0213 or infected and infected with siRNA: ${ }^{\#} P<0.05,{ }^{\# \#} P<0.01$, and ${ }^{\# \# \#} P<0.001$.

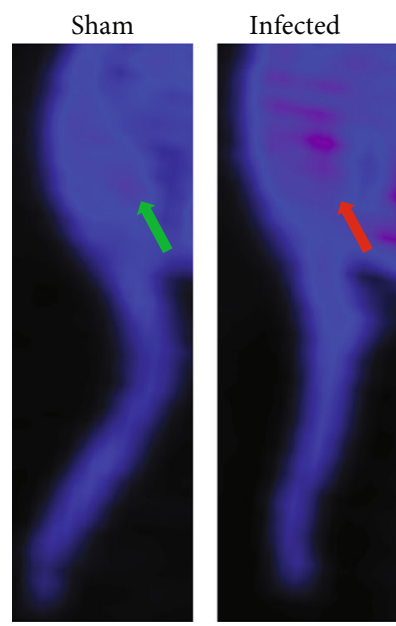

(a)

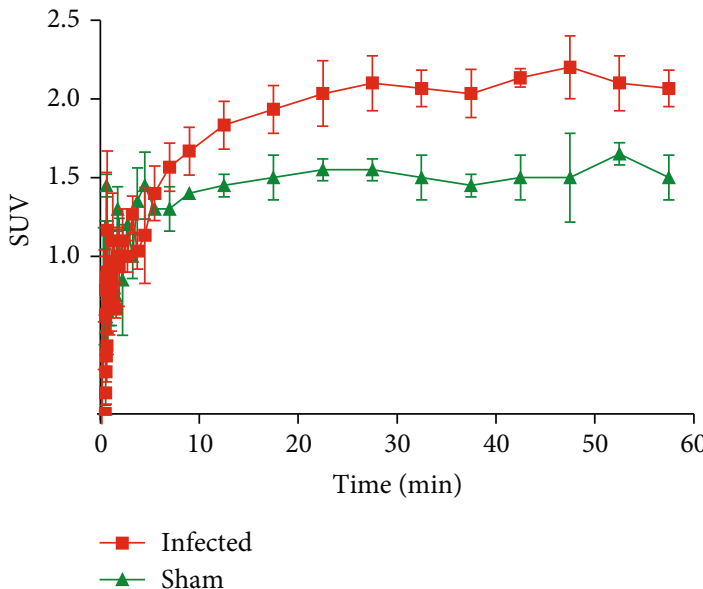

(b)

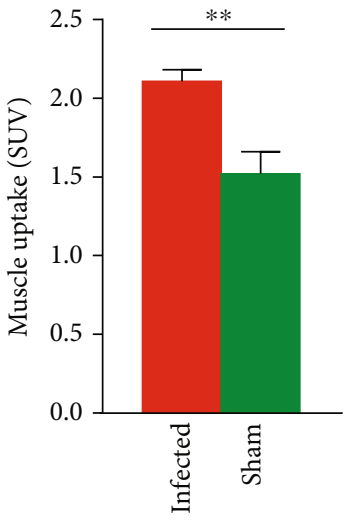

(c)

FIGURE 2: MicroPET imaging of S1PR1 activity in $S$ aureus-infected mice. (a) Representative sagittal microPET images of $\left[{ }^{18} \mathrm{~F}\right] \mathrm{TZ} 4877$ in the hind limb of mice. The tracer uptake was relatively low in the hind limb muscle with a SUV of $\sim 1.5$ in sham mice. Comparing with sham mice, the tracer uptake was significantly higher in the hind limb of infected mice; (b) time-activity curves showed that the tracer uptake in infected mice was significantly higher than sham mice; (c) the average tracer uptake in the hind limb muscle from 30 to 50 min of the PET scan showed a $\sim 39 \%$ increase of SUV in infected mice with a $P$ value of 0.0082 . Data represent the mean \pm SEM, $n=3$ for each group.

responses within 24 hours [39, 40]; the abscess lesions can increase over 5 to 7 days after infection [40] and can be gradually revolved over 7 to 9 days $[39,60]$.

In this study, we report a change of S1PR1 expression in response to the local $S$. aureus after 24 hours of infection. We observed a local change of $\left[{ }^{18} \mathrm{~F}\right] \mathrm{TZ} 4877$ uptake at the bacterial infection site as well as increased uptake of $\left[{ }^{18} \mathrm{~F}\right] \mathrm{TZ} 4877$ throughout the body. In general, both biodistribution and microPET studies showed that $\left[{ }^{18} \mathrm{~F}\right] \mathrm{TZ} 4877$ had high uptake in the majority of tested organs indicating that PET $\left[{ }^{18} \mathrm{~F}\right] \mathrm{TZ} 4877$ could be a reliable tool for quantifying S1PR1 in vivo. For example, at $30 \mathrm{~min}$ postradiotracer injection, the biodistribution data showed that the liver uptake was $\sim 15.27$ (\%ID/g); microPET studies showed that the liver uptake was $\sim 11.40$ (SUV). Notably, $\left[{ }^{18} \mathrm{~F}\right]$ TZ4877 entered the brain well with an SUV value of $\sim 3.57$ at $30 \mathrm{~min}$ posttracer injection, indicating that the tracer can penetrate the blood-brain barrier well and has a great potential for imaging S1PR1 in the central nervous system. 
TABLE 3: PET measurements of S1PR1-specific $\left[{ }^{18} \mathrm{~F}\right] \mathrm{TZ} 4877$ in $S$ aureus-infected and sham mice.

\begin{tabular}{lcccc}
\hline & Infected $^{*}$ & Sham $^{*}$ & $P$ value $^{\#}$ & Changes $\left.^{*} \%\right)$ \\
\hline Hind limb muscle & $2.11 \pm 0.04$ & $1.52 \pm 0.07$ & $=0.0001$ & $38.8 \%$ \\
Brain & $3.57 \pm 0.12$ & $2.65 \pm 0.14$ & $=0.0082$ & $34.8 \%$ \\
Liver & $11.40 \pm 0.20$ & $10.13 \pm 0.12$ & $<0.0001$ & $12.5 \%$ \\
\hline
\end{tabular}

${ }^{*} N=3$ per group, mean \pm SEM of average SUV from 30 to $50 \mathrm{~min}$. ${ }^{*}$ Two-tailed paired $t$-test.

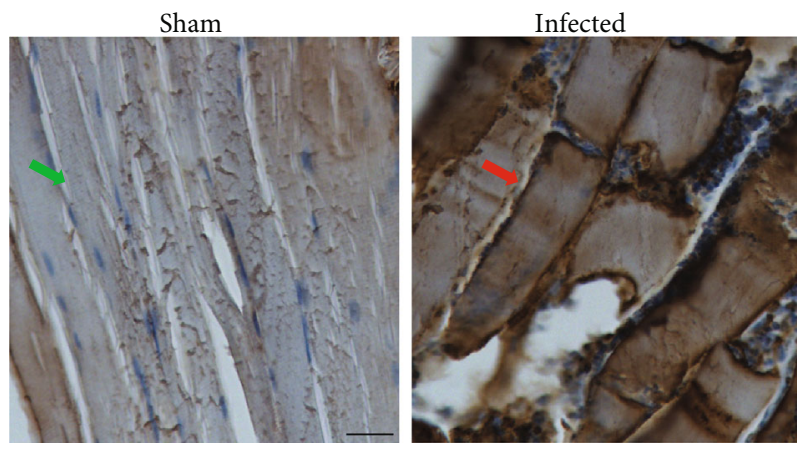

FIGURE 3: Immunohistochemistry analysis of S1PR1 in hind limb muscle of sham and $S$ aureus-infected mice. S1PR1 was significantly upregulated in the muscle of infected mice (red arrow) comparing with sham mice (green arrow), scale bar $=100 \mu \mathrm{m}$.

One of the major findings of this study is the upregulation of S1PR1 measured by $\left[{ }^{18} \mathrm{~F}\right]$ TZ4877 throughout the body in response to infection. Remarkably, this infectioninduced upregulation of S1PR1 showed an S. aureus dosedependent manner. S1PR1 is an immune modulator and plays a crucial role in the regulation of cytokine during inflammation [59]. It is reported that S1PR1 regulates cytokine production and host innate immune responses to pathogen infection [24]. In fact, several studies demonstrated that S1PR1-specific ligands can downregulate and control the massive innate inflammatory response, and endogenous S1P-S1PR1 axis could be a negative regulator of cytokine production $[25,59,61]$. The mechanisms of S1PR1 in the regulation of immune responses in response to pathogen infection remain not clear; our microPET study demonstrates a systemic activation of S1PR1 in response to local $S$. aureus infection and provides an evidence of the role of S1PR1 in the innate inflammatory response. In agreement with our microPET study, biodistribution data also showed a statistically increased uptake of $\left[{ }^{18} \mathrm{~F}\right] \mathrm{TZ} 4877$ in liver, kidney, and other tissues. In fact, accumulated evidence suggests that S1PR1 activation and signaling are impaired during infectious disease. For example, previous studies report that influenza infection can alter the expression level of S1PR1 in the liver, spleen, kidney, and even heart [26]. Also, suppression of early innate immune responses through S1PR1 signaling can significantly reduce mortality during infection with influenza virus by suppressing the pathogeninduced excessive host immune response, in other words, "cytokine storm" $[59,62,63]$. Innate immune response plays as the first line of defense against infections. It is well known that S1P/S1PR pathways have modulatory effects in cytokine secretion of innate immune response. In particular, S1PR1 regulates cytokine secretion in various types of cells including dendritic cells, macrophages, $\mathrm{T}$ lymphocytes, epithelial cells, and endothelial cells [61]. It is suggested that targeting on S1P/SPRR may be an effective therapy for such cases where the host inflammatory response is a major component in the disease process [61]. Particularly, numerous studies have shown that acute lung injury is a common consequence of cytokine storm in Middle East respiratory syndrome (MERS) and SARS, as well as COVID-19. S1PR1 inhibitor analogs, such as AAL-R and RP-002, which have already shown a protective effect from the pathophysiological response during influenza infection, have been suggested as potential immunomodulators to suppress the cytokine storm. Our study identified a systemic "storm" of S1PR1 activation in the presence of pathogens; this finding may provide a new insight into the relationship between pathogen-induced S1PR1 activation and cytokine storm. However, it remains unclear whether the systemic activation of S1PR1 is the direct result of pathogen infection; further characterization is required to understand more about this global protein activation.

On the other hand, accumulated evidence also suggests a potential role of S1P/S1PR pathways in regulating sepsis [9-11, 23, 64]. It is hypothesized that the S1P/S1PR pathway impairs antimicrobial defense in the pathogenesis of sepsis. The major processes of sepsis include immunological stimulation, systemic inflammation, and coagulopathy; recent studies suggest that the S1P/S1PR1 pathway is involved in the hyperinflammatory phase in sepsis and regulates the excessive release of cytokine; activation of S1PR1 reduces the severe complications in sepsis [64]. In fact, S. aureus is a common cause of sepsis normally caused by the bacterial replication in blood. Intravenous inoculation of $5 \times 10^{7}$ to $5 \times 10^{8} \mathrm{CFU}$ S. aureus can cause the animal to develop septic shock within 48 hours [42]. Though a mouse model for $S$. aureus-induced sepsis is usually introduced intravenously with up to $5 \times 10^{8} \mathrm{CFU}$ S. aureus [3], in our case, a relatively long infection time was used; the infected animal is possibly septic 24-hour postinoculation particularly in mice with high-dose infection. Overall, our study provides a direct evidence of S1PR1 activation in response to pathogen infection; our microPET study demonstrates the feasibility of imaging the infection-induced activation of S1PR1 using the S1PR1 specific tracer $\left[{ }^{18} \mathrm{~F}\right] \mathrm{TZ} 4877$. Future studies with different doses of $S$. aureus and different infection times are needed to understand the precise mechanisms of the $S$. aureus infection-induced activation of S1PR1.

In addition to the systemic activation of S1PR1, using microPET study with $\left[{ }^{18} \mathrm{~F}\right] \mathrm{TZ} 4877$, we also observed a local 
upregulation of S1PR1 in the muscle of the infection site but not the contralateral side of the muscle indicating that such upregulation of S1PR1 is tightly correlated with pathogenderived inflammation. In fact, recent data suggest that bacterial infections may affect inflammatory processes in the vascular wall and atherosclerotic plaque progression in peripheral arteries [65]. S1P-S1PR1 axis is involved in host protective effects during the inflammation by maintaining vascular integrity [66]. It is believed that activation of S1PR1 in endothelial cells can maintain vascular integrity and prevent vascular leak during inflammatory response. In our case, the local upregulation of S1PR1 in an infected region could be related to the infection-triggered vascular leak and is the part of physiological compensatory response in the endothelial cells to prevent vascular leak during the inflammation. Moreover, S1P-S1PR1 axis also plays an important role in the control of skeletal muscle metabolism, atrophy, and regeneration. Previous studies reported that S1P-S1PR1 axis is involved in the migration of satellite cell in the injured local to improve skeletal muscle tissue repair and regeneration [67]; intramuscular S1PR1 is elevated in regenerating muscle fibers and mediates skeletal muscle mass and function [68]. The detected upregulation of S1PR1 in the infected region could be related to the changes of muscle metabolism and regeneration induced by pathogen infection; further investigation is needed to understand the precise role of S1PR1 in the muscle in response to pathogen infection. Although the uptake of $\left[{ }^{18} \mathrm{~F}\right] \mathrm{TZ} 4877$ is relatively low in the muscle, the uptake of $\left[{ }^{18} \mathrm{~F}\right] \mathrm{TZ} 4877$ in the muscle of infected mice was significantly higher than that in the muscle of the control mice. Due to the relatively lower muscle uptake even in the infected mice, it will be a challenge for PET imaging with $\left[{ }^{18} \mathrm{~F}\right] \mathrm{TZ} 4877$ for the precise assessment of S1PR1 changes in muscle in response to pathogen infection. However, PET imaging with $\left[{ }^{18} \mathrm{~F}\right] \mathrm{TZ} 4877$ could provide a noninvasive tool for detecting S1PR1 in response to infection in other organs, such as the spinal cord, brain, lung, or liver $[33,34]$.

One limitation of the current study is the use of PBS instead of heat-killed $S$. aureus in the contralateral side of infection as an internal control. Heat-killed S. aureus has been widely used as an internal control in a murine myositis model. In this study, we attempt to investigate the S1PR1 expression in response to $S$. aureus infection using our S1PR1-specific radioligand $\left[{ }^{18} \mathrm{~F}\right] \mathrm{TZ} 4877$. Previous studies have demonstrated that heat-killed $S$. aureus can induce a strong anti-inflammatory response via Toll-Like Receptor 2 (TLR2) pathway; it can induce the production of TNF- $\alpha$, IL-6, and IL-10, as well as other cytokines and chemokines [69-71]. On the other hand, S1P receptors including S1PR1 can attenuate the TLR2 pathway $[72,73]$. To date, the effect of heat-killed $S$. aureus on S1PR1 remains unclear. To minimize the potential effect of heat-killed $S$. aureus on S1PR1 expression, we used PBS as an internal control in this study. Sepsis is a systemic inflammatory disease resulting from pathogen infection and is associated with acute and chronic changes in the central nervous system, particularly at the blood-brain barrier (BBB) [74]. To detect the changes of S1PR1 expression in response to $S$. aureus infection, an animal was injected with $S$. aureus at doses of $1 \times 10^{6} \mathrm{CFU}$ and $1 \times 10^{8} \mathrm{CFU}$, and a microPET study was performed post-injection of $S$. aureus. Although the increased S1PR1 expression at the hind limb of the animal in response to $S$. aureus was confirmed by immunohistochemistry study, the reason that caused the increased brain uptake of $\left[{ }^{18} \mathrm{~F}\right]$ TZ4877 in response to $S$. aureus is not clear. The increased brain uptake of $\left[{ }^{18} \mathrm{~F}\right] \mathrm{TZ} 4877$ could be resulted from increased brain S1PR1 expression or caused by sepsis-induced $\mathrm{BBB}$ breakdown. In addition, the increased brain uptake of $\left[{ }^{18} \mathrm{~F}\right] \mathrm{TZ} 4877$ in response to $S$. aureus was based on the assumption that the injection dose of $S$. aureus and incubation time (24hrs) did not cause the sepsis. Further study using different doses of $S$. aureus and incubation times is necessary to understand the exact mechanisms of the elevation of S1PR1 in response to $S$. aureus infection.

\section{Conclusions}

In summary, we investigated the expression of S1PR1 in $S$. aureus-infected mice using both ex vivo biodistribution studies and microPET imaging; our data suggested that the infectioninduced systemic S1PR1 activation is tightly correlated with the early immune response to infection. The uptake of the S1PR1 radiotracer $\left[{ }^{18} \mathrm{~F}\right] \mathrm{TZ} 4877$ can be blocked by S1PR1specific antagonist and S1PR1-specific DsiRNA. Further characterization of the mechanisms of infection-induced systemic S1PR1 activation and translational investigation of S1PR1 functions in infectious diseases may lead to a new strategy for the diagnosis and the treatment of infectious diseases.

\section{Data Availability}

The data that support the findings of this study are available from the corresponding author upon request.

\section{Ethical Approval}

All animal related experiments were carried out following the recommendations in the Guide for the Care and Use of Laboratory Animals of the National Institutes of Health. All experimental procedures involving animals were performed according to guidelines established by the Washington University in St. Louis Institutional Animal Care and Use Committee (IACUC).

\section{Conflicts of Interest}

The authors have no conflict of interest to declare.

\section{Acknowledgments}

We would like to acknowledge Robert Dennet and Michael Nickels at Washington University Cyclotron Facility for $\mathrm{Na}\left[{ }^{18} \mathrm{~F}\right] / \mathrm{F}$ production. We especially would like to acknowledge Nicole Fettig, Amanda Klaas, and Lori Strong at Mallinckrodt Institute of Radiology Preclinical Imaging Facility for their technical assistance. We thank Lynne Jones for helpful discussions. This work is supported by the National Institutes of Health under projects NS075527, NS103957, NS103988, and EB025815. 


\section{Supplementary Materials}

MicroPET/CT images of S1PR1 activity in sham and $S$. aureus-infected mice are provided as supplementary files. Supplementary Fig 1: representative sagittal, coronal, and transverse PET/CT images of $\left[{ }^{18} \mathrm{~F}\right] \mathrm{TZ} 4877$ in sham, lowdose infected, and high-dose infected mice. Supplementary Fig 2: representative PET and PET/CT images of $\left[{ }^{18} \mathrm{~F}\right] \mathrm{TZ4877}$ in hind limb muscle of sham and $S$ aureusinfected mice. (Supplementary Materials)

\section{References}

[1] H. F. Wertheim, D. C. Melles, M. C. Vos et al., "The role of nasal carriage in_Staphylococcus aureus_infections," Lancet Infect Dis, vol. 5, no. 12, pp. 751-762, 2005.

[2] L. F. McCaig, L. C. McDonald, S. Mandal, and D. Jernigan, "Staphylococcus aureus-associated skin and soft tissue infections in ambulatory care," Emerging Infectious Diseases, vol. 12, no. 11, pp. 1715-1723, 2006.

[3] H. K. Kim, D. Missiakas, and O. Schneewind, "Mouse models for infectious diseases caused by _Staphylococcus aureus_," Journal of immunological methods, vol. 410, pp. 88-99, 2014.

[4] S. Y. C. Tong, J. S. Davis, E. Eichenberger, T. L. Holland, and V. G. Fowler Jr., "Staphylococcus aureus infections: epidemiology, pathophysiology, clinical manifestations, and management," Clinical Microbiology Reviews, vol. 28, no. 3, pp. 603-661, 2015.

[5] S. Igawa, J. E. Choi, Z. Wang et al., "Human Keratinocytes Use Sphingosine 1-Phosphate and its Receptors to Communicate _Staphylococcus aureus_ Invasion and Activate Host Defense," Journal of Investigative Dermatology, vol. 139, no. 8, pp. 1743-1752.e5, 2019.

[6] O. H. Lee, Y. M. Kim, Y. M. Lee et al., "Sphingosine 1phosphate induces angiogenesis: its angiogenic action and signaling mechanism in human umbilical vein endothelial cells," Biochem Biophys Res Commun, vol. 264, no. 3, pp. 743-750, 1999.

[7] G. Wang and E. Bieberich, "Sphingolipids in neurodegeneration (with focus on ceramide and S1P)," Advances in biological regulation, vol. 70, pp. 51-64, 2018.

[8] H. Chi, "Sphingosine-1-phosphate and immune regulation: trafficking and beyond," Trends Pharmacol Sci, vol. 32, no. 1, pp. 16-24, 2011.

[9] J. Hou, Q. Chen, K. Zhang et al., "Sphingosine 1-phosphate receptor 2 signaling suppresses macrophage phagocytosis and impairs host defense against sepsis," Anesthesiology, vol. 123, no. 2, pp. 409-422, 2015.

[10] B. S. Shea and S. M. Opal, "The role of S1PR3 in protection from bacterial sepsis," Am J Respir Crit Care Med, vol. 196, no. 12, pp. 1500-1502, 2017.

[11] A. Feng, A. D. Rice, Y. Zhang, G. T. Kelly, T. Zhou, and T. Wang, "S1PR1-associated molecular signature predicts survival in patients with sepsis," Shock, vol. 53, no. 3, pp. 284-292, 2020.

[12] M. Arish, A. Husein, M. Kashif, M. Saleem, Y. Akhter, and A. Rub, "Sphingosine-1-phosphate signaling: unraveling its role as a drug target against infectious diseases," Drug Discovery Today, vol. 21, no. 1, pp. 133-142, 2016.

[13] G. Giovannoni, C. Hawkes, J. Lechner-Scott, M. Levy, E. Waubant, and J. Gold, "The COVID-19 pandemic and the use of MS disease-modifying therapies," Mult Scler Relat Disord, vol. 39, article 102073, 2020.

[14] G. Mallucci, A. Zito, B. D. Fabbro, and R. Bergamaschi, "Asymptomatic SARS-CoV-2 infection in two patients with multiple sclerosis treated with fingolimod," Mult Scler Relat Disord, vol. 45, article 102414, 2020.

[15] L. Jin, W. R. Liu, M. X. Tian, J. Fan, and Y. H. Shi, "The SphKs/S1P/S1PR1 axis in immunity and cancer: more ore to be mined," World J Surg Oncol, vol. 14, no. 1, p. 131, 2016.

[16] M. Matloubian, C. G. Lo, G. Cinamon et al., "Lymphocyte egress from thymus and peripheral lymphoid organs is dependent on S1P receptor 1," Nature, vol. 427, no. 6972, pp. 355360, 2004.

[17] M. L. Allende, J. L. Dreier, S. Mandala, and R. L. Proia, "Expression of the Sphingosine 1-Phosphate Receptor, $\mathrm{S}_{1} \mathrm{P}_{1}$, on T-cells Controls Thymic Emigration," J Biol Chem, vol. 279, no. 15, pp. 15396-15401, 2004.

[18] L. G. Ledgerwood, G. Lal, N. Zhang et al., "The sphingosine 1-phosphate receptor 1 causes tissue retention by inhibiting the entry of peripheral tissue $\mathrm{T}$ lymphocytes into afferent lymphatics," Nature Immunology, vol. 9, no. 1, pp. 42-53, 2008.

[19] M. L. Allende, G. Tuymetova, B. G. Lee, E. Bonifacino, Y. P. $\mathrm{Wu}$, and R. L. Proia, "S1P1 receptor directs the release of immature B cells from bone marrow into blood," The Journal of experimental medicine, vol. 207, no. 5, pp. 1113-1124, 2010.

[20] M. Ishii, J. G. Egen, F. Klauschen et al., "Sphingosine-1phosphate mobilizes osteoclast precursors and regulates bone homeostasis," Nature, vol. 458, no. 7237, pp. 524528, 2009.

[21] A. Weigert, B. Weichand, and B. Brune, "S1P regulation of macrophage functions in the context of cancer," Anti-cancer agents in medicinal chemistry, vol. 11, no. 9, pp. 818-829, 2011.

[22] Y. Y. Lan, A. de Creus, B. L. Colvin et al., "The sphingosine-1phosphate receptor agonist FTY720 modulates dendritic cell trafficking in vivo," American journal of transplantation: official journal of the American Society of Transplantation and the American Society of Transplant Surgeons, vol. 5, no. 11, pp. 2649-2659, 2005.

[23] Z. Wang, C. R. Sims, N. K. Patil, N. Gokden, and P. R. Mayeux, "Pharmacologic targeting of sphingosine-1-phosphate receptor 1 improves the renal microcirculation during sepsis in the mouse," J Pharmacol Exp Ther, vol. 352, pp. 61-66, 2015.

[24] Y. Li, P. Xie, M. Sun et al., "S1PR1 expression correlates with inflammatory responses to Newcastle disease virus infection," Infect Genet Evol, vol. 37, pp. 37-42, 2016.

[25] J. R. Teijaro, K. B. Walsh, J. P. Long et al., "Protection of ferrets from pulmonary injury due to H1N1 2009 influenza virus infection: immunopathology tractable by sphingosine-1phosphate 1 receptor agonist therapy," Virology, vol. 452453, pp. 152-157, 2014.

[26] S. Tong, J. Tian, H. Wang et al., "H9N2 avian influenza infection altered expression pattern of sphiogosine-1-phosphate receptor 1 in BALB/c mice," Virology journal, vol. 10, p. 296, 2013.

[27] K. Graber, F. Khan, B. Glück et al., "The role of sphingosine-1phosphate signaling in HSV-1-infected human umbilical vein endothelial cells," Virus research, vol. 276, article 197835, 2020.

[28] N. I. Lorè, B. Sipione, G. He et al., "Collaborative cross mice yield genetic modifiers for Pseudomonas aeruginosa infection in human lung disease," mBio, vol. 11, no. 2, 2020. 
[29] J. C. Mudd, P. Murphy, M. Manion et al., "Impaired T-cell responses to sphingosine-1-phosphate in HIV-1 infected lymph nodes," Blood, vol. 121, no. 15, pp. 2914-2922, 2013.

[30] F. Naz and M. Arish, "Battling COVID-19 pandemic: sphingosine-1-phosphate analogs as an adjunctive therapy?," Frontiers in Immunology, vol. 11, pp. 1102-1102, 2020.

[31] H. Jin, H. Yang, H. Liu et al., "A promising carbon-11-labeled sphingosine-1-phosphate receptor 1-specific PET tracer for imaging vascular injury," J Nucl Cardiol, vol. 24, no. 2, pp. 558-570, 2017.

[32] H. Liu, H. Jin, X. Yue et al., "PET imaging study of S1PR1 expression in a rat model of multiple sclerosis," Molecular imaging and biology: MIB: the official publication of the Academy of Molecular Imaging, vol. 18, no. 5, pp. 724-732, 2016.

[33] Z. Luo, A. J. Rosenberg, H. Liu, J. Han, and Z. Tu, "Syntheses and _in vitro_evaluation of new S1PR1 compounds and initial evaluation of a lead F-18 radiotracer in rodents," Eur J Med Chem, vol. 150, pp. 796-808, 2018.

[34] Z. Luo, J. Han, H. Liu et al., "Syntheses and in vitro biological evaluation of S1PR1 ligands and PET studies of four F-18 labeled radiotracers in the brain of nonhuman primates," $\mathrm{Org}$ Biomol Chem, vol. 16, no. 47, pp. 9171-9184, 2018.

[35] H. Liu, Z. Luo, J. Gu et al., "In vivo characterization of four 18F-Labeled S1PR1 tracers for neuroinflammation," Molecular imaging and biology: MIB: the official publication of the Academy of Molecular Imaging, vol. 22, no. 5, pp. 1362-1369, 2020.

[36] M. A. Sellmyer, I. Lee, C. Hou et al., "Bacterial infection imaging with $[18 \mathrm{~F}]$ fluoropropyl-trimethoprim," Proceedings of the National Academy of Sciences of the United States of America, vol. 114, no. 31, pp. 8372-8377, 2017.

[37] G. Gowrishankar, M. Namavari, E. B. Jouannot et al., "Investigation of 6-[ $\left.{ }^{18} \mathrm{~F}\right]$-fluoromaltose as a novel PET tracer for imaging bacterial infection," PLoS One, vol. 9, no. 9, pp. e107951-e107951, 2014.

[38] E. A. Weinstein, A. A. Ordonez, V. P. DeMarco et al., "Imaging Enterobacteriaceae infection in vivo with 18Ffluorodeoxysorbitol positron emission tomography," Sci Transl Med, vol. 6, no. 259, article 259ra146, 2014.

[39] C. Bunce, L. Wheeler, G. Reed, J. Musser, and N. Barg, "Murine model of cutaneous infection with gram-positive cocci," Infect Immun, vol. 60, no. 7, pp. 2636-2640, 1992.

[40] N. Malachowa, S. D. Kobayashi, K. R. Braughton, and F. R. DeLeo, "Mouse model of Staphylococcus aureus skin infection," Methods in molecular biology (Clifton, NJ), vol. 1031, pp. 109-116, 2013.

[41] M. van Oosten, T. Schäfer, J. A. C. Gazendam et al., "Real-time _in vivo_imaging of invasive- and biomaterial-associated bacterial infections using fluorescently labelled vancomycin," Nature Communications, vol. 4, no. 1, p. 2584, 2013.

[42] A. G. Cheng, M. McAdow, H. K. Kim, T. Bae, D. M. Missiakas, and O. Schneewind, "Contribution of coagulases towards Staphylococcus aureus disease and protective immunity," PLoS Pathog, vol. 6, no. 8, article e1001036, 2010.

[43] J. Quancard, B. Bollbuck, P. Janser et al., "A Potent and Selective $\mathrm{S}_{1} \mathrm{P}_{1}$ Antagonist with Efficacy in Experimental Autoimmune Encephalomyelitis," Chemistry \& Biology, vol. 19, no. 9, pp. 1142-1151, 2012.

[44] D. Yamane, M. A. Zahoor, Y. M. Mohamed et al., "Inhibition of Sphingosine Kinase by Bovine Viral Diarrhea Virus NS3 Is Crucial for Efficient Viral Replication and Cytopathogenesis,"
Journal of Biological Chemistry, vol. 284, no. 20, pp. 13648 13659, 2009.

[45] Y.-J. Seo, C. J. Pritzl, M. Vijayan et al., "Sphingosine kinase 1 serves as a pro-viral factor by regulating viral RNA synthesis and nuclear export of viral ribonucleoprotein complex upon influenza virus infection," PLoS One, vol. 8, no. 8, article e75005, 2013.

[46] N. J. Machesky, G. Zhang, B. Raghavan et al., "Human Cytomegalovirus Regulates Bioactive Sphingolipids," Journal of Biological Chemistry, vol. 283, no. 38, pp. 26148-26160, 2008.

[47] M. Vijayan, Y.-J. Seo, C. J. Pritzl, S. A. Squires, S. Alexander, and B. Hahm, "Sphingosine kinase 1 regulates measles virus replication," Virology, vol. 450-451, pp. 55-63, 2014.

[48] M. M. Monick, K. Cameron, L. S. Powers et al., "Sphingosine kinase mediates activation of extracellular signal-related kinase and Akt by respiratory syncytial virus," American journal of respiratory cell and molecular biology, vol. 30, no. 6, pp. 844-852, 2004.

[49] J. M. Carr, T. Kua, J. N. Clarke et al., "Reduced sphingosine kinase 1 activity in dengue virus type- 2 infected cells can be mediated by the $3^{\prime}$ untranslated region of dengue virus type-2 RNA," J Gen Virol, vol. 94, no. 11, pp. 2437-2448, 2013.

[50] A. L. St John, W. X. G. Ang, M.-N. Huang et al., "S1P-Dependent Trafficking of Intracellular_Yersinia pestis_through Lymph Nodes Establishes Buboes and Systemic Infection," Immunity, vol. 41, no. 3, pp. 440-450, 2014.

[51] A. Oizumi, H. Nakayama, N. Okino et al., "Pseudomonasderived ceramidase induces production of inflammatory mediators from human keratinocytes via sphingosine-1phosphate," PLoS One, vol. 9, no. 2, article e89402, 2014.

[52] Z. A. Malik, C. R. Thompson, S. Hashimi, B. Porter, S. S. Iyer, and D. J. Kusner, "Cutting Edge:Mycobacterium tuberculosisBlocks Ca2+Signaling and phagosome maturation in human macrophages via specific inhibition of sphingosine kinase," The Journal of Immunology, vol. 170, no. 6, pp. 2811-2815, 2003.

[53] H. Prakash, A. Lüth, N. Grinkina et al., "Sphingosine kinase-1 (SphK-1) regulates Mycobacterium smegmatis infection in macrophages," PLoS One, vol. 5, no. 5, article e10657, 2010.

[54] S. Ochi, M. Oda, H. Matsuda, S. Ikari, and J. Sakurai, “_Clostridium perfringens_ $\alpha$-Toxin Activates the Sphingomyelin Metabolism System in Sheep Erythrocytes," Journal of Biological Chemistry, vol. 279, no. 13, pp. 12181-12189, 2004.

[55] M. Yadav, L. Clark, and J. S. Schorey, "Macrophage's proinflammatory response to a mycobacterial infection is dependent on sphingosine kinase-mediated activation of phosphatidylinositol phospholipase C, protein kinase C, ERK1/2, and phosphatidylinositol 3-kinase," The Journal of Immunology, vol. 176, pp. 5494-5503, 2006.

[56] A. Lepletier, L. de Almeida, L. Santos et al., "Early doublenegative thymocyte export in Trypanosoma cruzi infection is restricted by sphingosine receptors and associated with human Chagas disease," PLoS Neglected Tropical Diseases, vol. 8, no. 10, article e3203, 2014.

[57] T. McQuiston, C. Luberto, and M. Del Poeta, "Role of host sphingosine kinase 1 in the lung response against cryptococcosis," Infection and Immunity, vol. 78, pp. 2342-2352, 2010. 
[58] T. McQuiston, C. Luberto, and M. Del Poeta, "Role of sphingosine-1-phosphate $(\mathrm{S} 1 \mathrm{P})$ and S1P receptor 2 in the phagocytosis of Cryptococcus neoformans by alveolar macrophages," Microbiology (Reading), vol. 157, pp. 1416-1427, 2011.

[59] J. R. Teijaro, K. B. Walsh, S. Cahalan et al., "Endothelial cells are central orchestrators of cytokine amplification during influenza virus infection," Cell, vol. 146, no. 6, pp. 980-991, 2011.

[60] A. D. Kennedy, J. B. Wardenburg, D. J. Gardner et al., "Targeting of alpha-hemolysin by active or passive immunization decreases severity of USA300 skin infection in a mouse model," The Journal of infectious diseases, vol. 202, no. 7, pp. 1050-1058, 2010.

[61] K. B. Walsh, J. R. Teijaro, H. Rosen, and M. B. A. Oldstone, "Quelling the storm: utilization of sphingosine-1-phosphate receptor signaling to ameliorate influenza virus-induced cytokine storm," Immunol Res, vol. 51, no. 1, pp. 15-25, 2011.

[62] D. Marsolais, B. Hahm, K. H. Edelmann et al., "Local not systemic modulation of dendritic cell S1P receptors in lung blunts virus-specific immune responses to influenza," Mol Pharmacol, vol. 74, no. 3, pp. 896-903, 2008.

[63] D. Marsolais, B. Hahm, K. B. Walsh et al., "A critical role for the sphingosine analog AAL- $\mathrm{R}$ in dampening the cytokine response during influenza virus infection," Proceedings of the National Academy of Sciences of the United States of America, vol. 106, no. 5, pp. 1560-1565, 2009.

[64] M. S. Winkler, A. Nierhaus, M. Holzmann et al., "Decreased serum concentrations of sphingosine-1-phosphate in sepsis," Critical Care, vol. 19, no. 1, pp. 372-372, 2015.

[65] J. Budzyński, J. Wiśniewska, M. Ciecierski, and A. Kędzia, "Association between bacterial infection and peripheral vascular disease: a review," Int J Angiol, vol. 25, no. 1, pp. 3-13, 2016.

[66] E. Camerer, J. B. Regard, I. Cornelissen et al., "Sphingosine-1phosphate in the plasma compartment regulates basal and inflammation-induced vascular leak in mice," The Journal of clinical investigation, vol. 119, pp. 1871-1879, 2009.

[67] M. Fortier, N. Figeac, R. B. White, P. Knopp, and P. S. Zammit, "Sphingosine-1-phosphate receptor 3 influences cell cycle progression in muscle satellite cells," Developmental biology, vol. 382, no. 2, pp. 504-516, 2013.

[68] N. Ieronimakis, M. Pantoja, A. L. Hays et al., "Increased sphingosine-1-phosphate improves muscle regeneration in acutely injured mdx mice," Skeletal Muscle, vol. 3, p. 20, 2013.

[69] O. M. Peck, D. L. Williams, K. F. Breuel et al., "Differential regulation of cytokine and chemokine production in lipopolysaccharide-induced tolerance and priming," Cytokine, vol. 26, pp. 202-208, 2004.

[70] O. Takeuchi, K. Hoshino, and S. Akira, "Cutting edge: TLR2deficient and MyD88-deficient mice are highly susceptible to $<$ em $>$ Staphylococcus aureus $</$ em $>$ infection," The Journal of Immunology, vol. 165, pp. 5392-5396, 2000.

[71] A. Lembo, C. Kalis, C. J. Kirschning et al., "Differential contribution of Toll-like receptors 4 and 2 to the cytokine response toSalmonella entericaSerovar Typhimurium andStaphylococcus aureusin mice," Infection and Immunity, vol. 71, no. 10, pp. 6058-6062, 2003.

[72] A. I. Duenas, M. Aceves, I. Fernandez-Pisonero et al., "Selective attenuation of Toll-like receptor 2 signalling may explain the atheroprotective effect of sphingosine 1-phosphate," Cardiovascular Research, vol. 79, no. 3, pp. 537-544, 2008.
[73] S. Hamidi, M. Schäfer-Korting, and G. Weindl, "TLR2/1 and sphingosine 1-phosphate modulate inflammation, myofibroblast differentiation and cell migration in fibroblasts," Biochim Biophys Acta, vol. 1841, pp. 484-494, 2014.

[74] T. Barichello, J. S. Generoso, A. Collodel, F. Petronilho, and F. Dal-Pizzol, "The blood-brain barrier dysfunction in sepsis," Tissue Barriers, vol. 9, no. 1, article 1840912, 2021. 